\title{
Resonant inelastic soft $x$-ray scattering of CdS: a two-dimensional electronic structure map approach
}

L. Weinhardt ${ }^{1,2 *}$, O. Fuchs ${ }^{2}$, A. Fleszar ${ }^{3}$, M. Bär ${ }^{1}$, M. Blum ${ }^{1,2}$, M. Weigand ${ }^{2}$, J.D. Denlinger ${ }^{4}$, W. Yang $^{4}$, W. Hanke ${ }^{3}$, E. Umbach ${ }^{2,5}$, and C. Heske ${ }^{1, * *}$

${ }^{1}$ Department of Chemistry, University of Nevada, Las Vegas, NV 89154-4003, USA

${ }^{2}$ Universität Würzburg, Experimentelle Physik II, Am Hubland, 97074 Würzburg, Germany

${ }^{3}$ Universität Würzburg, Theoretische Physik I, Am Hubland, 97074 Würzburg, Germany

${ }^{4}$ Advanced Light Source, Lawrence Berkeley National Laboratory, Berkeley, CA 94720, USA

${ }^{5}$ FZ Karlsruhe, Hermann-von-Helmholtz-Platz 1, 76344 Eggenstein-Leopoldshafen, Germany

Resonant inelastic x-ray scattering (RIXS) with soft x-rays is uniquely suited to study the electronic structure of a variety of materials, but is currently limited by low (fluorescence yield) count rates. This limitation is overcome with a new high-transmission spectrometer that allows to measure soft x-ray RIXS “maps”. The S $\mathrm{L}_{2,3}$ RIXS map of CdS is discussed and compared with density functional calculations. The map allows the extraction of decay channel-specific "absorption spectra", giving detailed insight into the wave functions of occupied and unoccupied electronic states.

PACS numbers: 78.70.En, 78-70.Dm, 71.15.Mb

*corresponding author: lothar.weinhardt@physik.uni-wuerzburg.de

** Electronic mail: heske@unlv.nevada.edu 


\section{Introduction}

Since the first observation of resonant effects in soft x-ray fluorescence [1], resonant inelastic xray scattering (RIXS [2]) with soft X-rays has been successfully developed as a method to investigate the band structure of solids [3-12]. Recently, RIXS was also used to study the electronic structure of liquids (e.g., water [13-15]). RIXS is a local technique: a wave function overlap between decaying valence electron and the spatially confined core hole is necessary, which gives information about the local distribution of the valence wave function with respect to the core hole wave function.

Due to the very small soft x-ray fluorescence yield and the small solid angle of high-resolution spectrometers, RIXS is a "low-countrate" technique. To date, this limits a RIXS series to a small number of spectra with selected excitation energies around the investigated absorption edge. We have recently developed a high-resolution, high-transmission soft x-ray spectrometer [16], which now allows recording complete RIXS "maps" in short time ( $<1$ hour). These "maps" display a two-dimensional intensity plot as a function of excitation and emission energy. They hence contain entire RIXS spectra for each data point in a conventional $\mathrm{x}$-ray absorption spectroscopy (XAS) scan as well as decay channel-specific absorption spectra. We note that Agåker et al. [17] presented soft x-ray emission data in a similar way, however with a much wider step size and lower singal-to-noise ratio.

In this paper, we use the $\mathrm{S}_{2,3}$ emission of $\mathrm{CdS}$ to demonstrate the strengths of the RIXS map approach. CdS is one of the most important II-VI semiconductors and is used in various applications (e.g., thin-film solar cells [18,19], nanoparticles [20-22], and light-emitting diodes [23]). We show that the RIXS map approach substantially improves the extraction of band structure information which is essential for an insight-based optimization of CdS-based devices in all of 
these applications. Especially if resonant effects are weak and non-resonant contributions dominate (as is the case for CdS [24]) the dense two-dimensional plot makes it much easier to identify dispersing features containing band-structure information. Furthermore, the possibility to generate decay channel-specific XAS spectra opens a new pathway to information about the local (spatial) distribution of the occupied and unoccupied states by comparing the map with density functional theory (DFT) calculations. Thus, we are able to separate $\mathrm{L}_{2}$ and $\mathrm{L}_{3}$ absorption spectra in spite of a spin-orbit-splitting of only $1.2 \mathrm{eV}$.

\section{Experimental and Theoretical Details}

RIXS maps were recorded at Beamline 8.0.2 of the Advanced Light Source, Lawrence Berkeley National Laboratory, using a $\mathrm{CdS}(0001)$ single crystal (Mateck). The x-ray spectrometer is based on an entrance slit-less design, a spherical collecting mirror, a variable line spacing grating, and a CCD detector [16]. The beam spot was $\sim 20 \mu \mathrm{m}$ in the dispersive direction of the spectrometer resulting in a resolving power of $>1000$. The beamline resolution was set to $<150 \mathrm{meV}$ at the $\mathrm{S}$ $\mathrm{L}_{2,3}$ edge. With these settings, the transmission of our new x-ray spectrometer is about two orders of magnitude higher than conventional x-ray spectrometers. This could be estimated based on a direct comparison (i.e., identical beamline parameters and flux) with the permanently installed SXF spectrometer at beamline 8.0 [25]. The latter is a spherical grating spectrometer in Rowland geometry and is arguably one of the best soft x-ray spectrometers in the world. Emission and absorption energy scales were calibrated in accordance with our earlier measurements on CdS [24]. The spectra within the map were normalized to the incoming photon flux which was measured with a gold mesh situated downstream of the last mirror of the beamline. An excitation energy step size of $0.1 \mathrm{eV}$ was chosen. 
Theoretical RIXS maps were calculated based on the Kramers-Heisenberg formalism and density functional theory using the local density approximation (for more details see [24]).

\section{Results and Discussion}

To demonstrate the strength of the RIXS map approach, CdS is a particularly suitable example since its $\mathrm{S} \mathrm{L}_{2,3}$ spectrum consists of several well-separated features that can be attributed to different decay channels $[24,26]$. As reported previously, band structure information is difficult to obtain from "conventional" CdS RIXS, since the spectra are dominated by incoherent contributions, giving rise to only small resonant effects in the spectra [24]. The $\mathrm{S} \mathrm{L}_{2,3}$ map of CdS, recorded in 33 minutes, is shown in Fig. 1. Above the map, the non-resonant x-ray emission spectrum (excited at $175.3 \mathrm{eV}$ ) is plotted. The $\mathrm{S}_{2,3}$ emission spectrum consists of transitions from the S 3s-derived band (in the following "3s emission") around $148 \mathrm{eV}$, from $\mathrm{Cd} 4 \mathrm{~d}-$ derived bands ("Cd $4 \mathrm{~d}$ emission") at $150.7 \mathrm{eV}$ and $151.9 \mathrm{eV}$, and from the upper valence band ("UVB emission", $154 \mathrm{eV}$ to $160 \mathrm{eV}$ ), into $2 \mathrm{p}$ core holes [26-28]. Each of these transitions forms a doublet because of the spin-orbit splitting (1.2 eV [28]) of the $\mathrm{S} 2 \mathrm{p}$ core hole, which is clearly visible in the sharp $\mathrm{Cd} 4 \mathrm{~d}$ emission lines. In addition, we find a strong elastically scattered Rayleigh line at the position of the excitation energy $(175.3 \mathrm{eV})$ and a broad feature around $191 \mathrm{eV}$ that can be related to the emission of visible light. This visible light is emitted from the excitation spot on the sample and is (indirectly) reflected onto the detector (note that the energetic position of this feature is not correlated to the real energy of these photons).

On the right hand side of the map in Fig. 1, the integrated intensity of the emission spectra (between $133 \mathrm{eV}$ and $159 \mathrm{eV}$, i.e., excluding Rayleigh line and visible light) is plotted as function of 
excitation energy. This spectrum represents the (remarkably smooth) partial fluorescence yield XAS spectrum which agrees well with previously published data [24,26]. The Rayleigh line is found as a diagonal line in the middle of the map, representing the energy of the exciting photons. On the low-energy side of this feature, the $\mathrm{S} \mathrm{L}_{2,3}$ emission spectrum of $\mathrm{CdS}$ is found, while the high-energy side is dominated by the visible light signal. (A very weak feature associated with the $\mathrm{Cd} \mathrm{M}_{4,5}$ emission, excited and detected in higher orders, is also present at about $170 \mathrm{eV}$.)

In Fig. 2 (top) the calculated RIXS map of CdS is shown on an expanded photon energy scale. The calculation includes experimental (Gaussian) broadening, but no further broadening due to lifetime effects. We find that the S $3 \mathrm{~s}$ and $\mathrm{Cd} 4 \mathrm{~d}$ emissions (left) exhibit no shift in energy since the dispersion of the corresponding bands is weak. In contrast, directly above the absorption edge the UVB emission (right) shifts to lower emission energies with increasing excitation energy. This shift, which reappears at the $\mathrm{L}_{2}$ edge, can be explained by the downward dispersion of the uppermost valence band and the excitation energy-dependent shift of the excitation/emission process away from the $\Gamma$-point $[24]$.

This shift can also easily be identified in the (magnified) experimental map (Fig. 2, bottom) most obviously as a shift of the high energy edge of the valence band emission. Note that the experimental spectra are a sum of resonant (coherent) and non-resonant (incoherent) contributions and are dominated by the latter. In fact, in an earlier work by Zhou et al., no resonant changes in the $\mathrm{L}_{2,3}$ emission spectra of CdS could be identified at all [26]. This leads to the observation that in the experimental data the $\mathrm{Cd} 4 \mathrm{~d}$ emission and the low energy part of the UVB emission already appear at lower excitation energies than in the calculation which only represents the resonant 
(coherent) emission. This can also be clearly seen by the higher intensity near the onset of the experimental absorption scans compared to the calculated ones as will be discussed below. The unambiguous presence of dispersing features in the present experimental data demonstrates the power of the map approach to identify weak resonant features. By generating a contour plot of the calculated map and plotting it on top of the experimental map (see Fig. 2), we can now directly compare theory and experiment. We find a very good agreement of positions and intensity variations of the various features over the entire excitation energy range, validating the calculated band structure. In fact, this approach can be used to optimize the calculated band structure for less known materials to a degree that was previously impossible. Note that the theoretical map does not include the lifetime broadening that is present in the experiment (and that depends on the emission energy, i.e., that varies throughout the emission spectrum). Thus, the spectral features are more clearly seen in the calculated map as compared to the experimental.

The RIXS map approach also allows to studying the excitation energy-dependent intensity of selected decay channels. To date, partial fluorescence yield spectra are recorded with the total count rate from a selected energy window of an x-ray spectrometer. The RIXS map in Fig. 1 represents an absorption scan (with an XAS-typical excitation energy step size) in which a highresolution x-ray emission spectrum with good statistics is recorded at each excitation energy. This allows to deliberately choose specific spectral features to obtain an "absorption" scan for a particular decay channel (e.g., emission from a specific valence orbital/band). In the following, we will call such extracted spectra "absorption spectra" although such spectra are usually taken in an integrating mode. 
In the present case, we can easily separate $\mathrm{S} 3 \mathrm{~s}$ emission, $\mathrm{Cd} 4 \mathrm{~d}$ emission, and emission from the UVB. Furthermore, with the exception of the UVB emission, we can distinguish between $\mathrm{L}_{2}$ and $\mathrm{L}_{3}$ emission, enabling us to plot a separate $\mathrm{S}_{2}$ and $\mathrm{S}_{3}$ absorption spectrum over the whole energy range, despite a spin-orbit splitting of only $1.2 \mathrm{eV}$. The separation of the different features (S 3s and $\mathrm{Cd} 4 \mathrm{~d}$ emission peaks and their spin-orbit splitting) was performed as follows. Below the $\mathrm{L}_{2}$ edge, it is easy to separate $\mathrm{S} 3 \mathrm{~s}$ and $\mathrm{Cd} 4 \mathrm{~d}$ emission, because the $\mathrm{L}_{2}$ core hole is not yet "turned on" and the separation between the $\mathrm{L}_{3}$-derived $\mathrm{S} 3 \mathrm{~s}$ and $\mathrm{Cd} 4 \mathrm{~d}$ emission is sufficiently large and can be fitted as shown in Fig.3. For a more detailed decomposition of the $\mathrm{L}_{3}$ emission see Ref. 17. Assuming that the peak shapes of the $\mathrm{L}_{2}$ emission are very similar to those of the $\mathrm{L}_{3}$ emission (which is supported by our calculations), we have shifted the two $L_{3}$ components (i.e., the S $3 \mathrm{~s}$ and $\mathrm{Cd} 4 \mathrm{~d}$ features, respectively) by the spin-orbit splitting of $1.2 \mathrm{eV}$ to describe the $\mathrm{L}_{2}$ emission. Each spectrum was then fitted with these four (2x2) components using only the intensities as fit parameters. The resulting spectral decomposition is exemplarily shown for two different excitation energies in Fig. 3. The derived peak areas are used in the following to compile the decay channel-specific absorption spectra (called "S $3 \mathrm{~s}$ " and "Cd $4 \mathrm{~d}$ " in the following). For the UVB emission, no separation into $L_{2}$ and $L_{3}$ is possible due to the presence of the dispersive features. Therefore, the (overall) fluorescence yield of the UVB emission was determined by integrating between emission energies of $154 \mathrm{eV}$ and $161 \mathrm{eV}$ (called "UVB" in the following).

The experimental $L_{3}$ and $L_{2}$ absorption spectra are shown in Fig. 4 a). The dashed line (labeled $\mathrm{L}_{2}{ }^{*}$ ) represents the $\mathrm{L}_{2}$ spectrum shifted by $1.2 \mathrm{eV}$ (i.e., the spin-orbit splitting) to lower excitation energies and multiplied by a factor of 2 (to account for the multiplicity of $\mathrm{L}_{2}$ and $\mathrm{L}_{3}$ ). One would expect the absorption spectra of both edges to be equal (if the multiplicities are taken into account). Indeed, the direct comparison between $\mathrm{L}_{2}{ }^{*}$ and $\mathrm{L}_{3}$ qualitatively shows the same features in 
both spectra. However, there are significant quantitative differences. For example, the absorption edge (labeled A) is weaker for $\mathrm{L}_{2}{ }^{*}$, and is slightly shifted to higher energies. To understand the quantitative differences, the "competition" between $\mathrm{L}_{2}$ and $\mathrm{L}_{3}$ absorption has to be taken into account. Wherever strong $\mathrm{L}_{3}$ absorption occurs, less photons are available for the $\mathrm{L}_{2}$ absorption and vice versa. Since the $\mathrm{L}_{3}$ absorption is about twice as strong, its impact on the $\mathrm{L}_{2}$ absorption is stronger than the impact of $\mathrm{L}_{2}$ on $\mathrm{L}_{3}$. A close look at the spectra shows that feature $\mathrm{A}$ for $\mathrm{L}_{3}$ has its maximum at the absorption onset of $\mathrm{L}_{2}$, explaining the weaker and delayed absorption onset in the $\mathrm{L}_{2}$ absorption. Similarly, feature B appears weaker and with a maximum at lower energies for $\mathrm{L}_{2}{ }^{*}$ than for $\mathrm{L}_{3}$, because feature $\mathrm{C}$ for $\mathrm{L}_{3}$ appears at the high-energy side of feature $\mathrm{B}$ of $\mathrm{L}_{2}$, reducing its intensity and shifting the maximum to lower energies. This also leads to the fact that feature $\mathrm{B}$ of $\mathrm{L}_{2}{ }^{*}$ is narrower than feature $\mathrm{B}$ of $\mathrm{L}_{3}$. All other differences between the two absorption spectra can be explained in a similar way.

In Fig. 4 b), the $\mathrm{L}_{2,3}$ absorption spectra of the different decay channels ("S $3 \mathrm{~s}$ ", "Cd $4 d$ ", and "UVB") are plotted versus excitation energy (after edge-jump normalization). We find very similar spectra for the three decay channels, except for a significant difference close to the absorption onset, which is observed for the first time and explained in the following. The RIXS intensity (described by the Kramers-Heisenberg formalism) depends on matrix elements containing the ground state, the intermediate state (i.e., a core hole and an electron in the conduction band), and the final state (i.e., a valence hole and an electron in the conduction band). Since the initial state and the ensemble of intermediate states are the same for all decay channels, the observed differences give insight into the transition matrix elements between intermediate and final states and thus into the wave functions of the unoccupied and occupied states (taking the operator describing the emitted photon into account). Indeed, our calculations in Fig. 4 c) similarly show a strong 
emission from the upper valence band and a weak $\mathrm{Cd} 4 \mathrm{~d}$ emission near the absorption onset. Note that the calculated spectra have a smaller intensity close to the absorption edge due to the presence of a core exciton in the experiment [24, 29], which is not included in the calculation. For a direct comparison between theory and experiment, we have removed the spectral impact of the core exciton, by calculating difference spectra in which the absorption spectrum for the $\mathrm{S} 3 \mathrm{~s}$ decay channel was subtracted from those of the UVB and $\mathrm{Cd} 4 \mathrm{~d}$ emission, respectively (note that this approach assumes that the spectral contribution of the core exciton is independent of the decay channel in which it is observed). The good agreement of the experimental (solid) and theoretical (dashed) difference spectra near the absorption onset (Fig. 4d) validates the calculated wave functions and thus gives detailed local (spatial) information about the wave functions of the occupied valence and unoccupied conduction band states.

The visible light emission caused by the x-ray excitation of the sample also shows an interesting behavior. As apparent from Fig. 4 e), it is proportional to the inverse $\mathrm{S} \mathrm{L}_{2,3}$ absorption. This is explained as follows: Below the $\mathrm{S}_{2,3}$ absorption edge, the exciting x-rays penetrate deep into the bulk, creating only $\mathrm{Cd} 4 \mathrm{~s}, \mathrm{Cd} 4 \mathrm{p}$, and valence holes. Decay cascades of these holes, as well as decay and reabsorption processes of the photoexcited electrons lead to fluorescence in the visible range. Once the excitation energy is high enough to excite S $2 p$ electrons, the attenuation length of the x-rays is strongly reduced (from 0.4 to $0.1 \mu \mathrm{m}$ [30]). This reduces the probability of the mentioned decay processes because a larger fraction of the photoexcited electrons is produced closer to the surface. Effectively, the creation of valence holes is reduced, and hence the intensity of the visible fluorescence, which, in consequence, shows a minimum at all photon energies at which the $\mathrm{S}_{2,3}$ absorption shows a maximum. 


\section{Summary}

We have presented a full resonant inelastic soft x-ray scattering map of CdS. We have demonstrated the advantage of the map approach when identifying even weak band structure-related features in very good agreement with our theoretical maps. For CdS, the RIXS maps also allow (a) the separation of the $\mathrm{S} \mathrm{L}_{2}$ and $\mathrm{L}_{3}$ absorption spectra and (b) the extraction of decay channelspecific absorption spectra. These give insight into the wave functions of unoccupied states with respect to the decaying valence states. The RIXS map concept thus gives valuable detailed insight into the electronic structure of a variety of material systems that is not attainable with any other technique.

\section{Acknowledgements}

This work was supported by the German BMBF (project No. 05KS4WWA/6), the DFG through SFB 410 (TP B4), the National Renewable Energy Lab (subcontract XXL-5-44205-12), the

DFG Emmy Noether program (M. Bär), and by the U.S. Dept. of Energy at the ALS under grant no. DE-AC02-05CH11231. The calculations were performed at the ZAM Jülich. 


\section{References:}

[1] J.-E. Rubensson et al., Phys. Rev. Lett. 60, 1759 (1988).

[2] Note that RIXS is also often called "resonant soft x-ray emission", "resonant x-ray emission spectroscopy", or "resonant soft x-ray fluorescence".

[3] J.-E. Rubensson et al., Phys. Rev. Lett. 64, 1047 (1990).

[4] Y. Ma et al., Phys. Rev. Lett. 69, 2598 (1992).

[5] P.D. Johnson and Y. Ma, Phys. Rev. B 49, 5024 (1994).

[6] Y. Ma, Phys. Rev. B 49, 5799 (1994).

[7] J.A. Carlisle et al., Phys. Rev. Lett. 74, 1234 (1995).

[8] J. Lüning et al., Phys. Rev. B 56, 13147 (1997).

[9] E.L. Shirley, Phys. Rev. Lett. 80, 794 (1998).

[10] J.A. Carlisle et al., Phys. Rev. B 59, 7433 (1999).

[11] S. Eisebitt and W. Eberhardt, J. Electron Spectrosc. Rel. Phenom. 110, 335 (2000).

[12] D. Eich et al., Phys. Rev. B 73, 115212 (2006).

[13] J.-H. Guo et al., Phys. Rev. Lett. 89, 137402 (2002), J.-H. Guo et al., Phys. Rev. Lett. 91, $157401(2003)$.

[14] M. Odelius et al., Phys. Rev. Lett. 94, 227401 (2005).

[15] O. Fuchs et al., Phys. Rev. Lett. 100, 027801 (2008).

[16] O. Fuchs et al., in preparation.

[17] M. Agåker, T. Käämbre, C. Glover, T. Schmitt, M. Mattesini, R. Ahuja, J. Söderström, and J.-E. Rubensson, Phys. Rev. B 73, 245111 (2006).

[18] C. Heske, D. Eich, R. Fink, E. Umbach, T. van Buuren, C. Bostedt, L. Terminello, S. Kakar, M. Grush, T. Callcott, et al., Appl. Phys. Lett. 74, 1451 (1999). 
[19] M. Morkel, L. Weinhardt, B. Lohmüller, C. Heske, E. Umbach, W. Riedl, S. Zweigart, and F. Karg, Appl. Phys. Lett. 79, 4482 (2001).

[20] M. Bruchez, M. Moronne, P. Gin, S. Weiss, and A.P. Alivisatos, Science 281, 2013 (1998).

[21] J. Lüning, J. Rockenberger, S. Eisebitt, J.-E. Rubensson, A. Karl, A. Kornowski, H. Weller, and W. Eberhardt, Solid State Commun. 112, 5 (1999).

[22] A.D. Dinsmore, D.S. Hsu, S.B. Qadri, J.O. Cross, T.A. Kennedy, H.F. Gray, and B.R. Ratna, J. Appl. Phys. 88, 4985 (2000).

[23] M. Schmidt, M. Grün, S. Petillon, E. Kurtz, and C. Klingshirn, Appl. Phys. Lett. 77, 85 (2000).

[24] L. Weinhardt et al., Phys. Rev. B 75, 165207 (2007).

[25] J. J. Jia, T. A. Callcott, J. Yurkas, A. W. Ellis, F. J. Himpsel, M. G. Samant, J. Stöhr, D. L. Ederer, J. A. Carlisle, E. A. Hudson, et al., Rev. Sci. Instrum. 66, 1394 (1995).

[26] L. Zhou et al., Phys. Rev. B 55, 5051 (1997).

[27] G. Eichhoff, Annalen der Physik 456, 55 (1958).

[28] S. Svensson et al., Phys. Rev. A 43, 6441 (1991).

[29] J. Lüning et al., Solid State Commun. 112, 5 (1999).

[30] http://henke.lbl.gov/optical_constants/atten2.html (Nov. 5, 2007). 


\section{Figure captions:}

FIG. 1. Experimental resonant inelastic soft x-ray scattering (RIXS) map of CdS. The emission intensity is color-coded (see color scale in Fig. 2) and given as a function of emission energy and excitation energy. The upper panel shows a non-resonant x-ray emission spectrum (excitation energy: $175.3 \mathrm{eV}$ ). The right panel shows an absorption spectrum with fluorescence yield detection, i.e., the $\mathrm{S} \mathrm{L}_{2,3}$ emission intensity (integrated between emission energies of $133 \mathrm{eV}$ and $159 \mathrm{eV}$ ) as a function of excitation energy.

FIG. 2. Top: Calculated RIXS map of CdS. Bottom: Contour lines of the calculated map and the corresponding experimental RIXS map of CdS.

FIG. 3. Fit of the experimental data with four spectral components, exemplarily shown at two excitation energies. Below each spectrum, the corresponding residuum is given.

FIG. 4. (a) Separated experimental $\mathrm{L}_{3}$ and $\mathrm{L}_{2}$ absorption spectra extracted from RIXS maps of $\mathrm{CdS}$ (solid lines). For comparison, the dashed line shows the magnified $(\times 2) \mathrm{L}_{2}$ spectrum after a $1.2 \mathrm{eV}$ shift $\left(\mathrm{L}_{2}{ }^{*}\right)$. (b) Separated experimental absorption spectra of different decay channels ("UVB" corresponds to the upper valence band emission intensity). (c) Calculated absorption spectra for the different decay channels. (d) Amplified difference spectra of the experimental spectra in b (shown as solid lines) and of the calculated spectra in c (dashed lines). (e) Energy dependence of the intensity of the emitted visible light. For comparison, the inverted $\mathrm{L}_{2,3}$ emission intensity is shown as a dashed line (on an arbitrary intensity scale). 


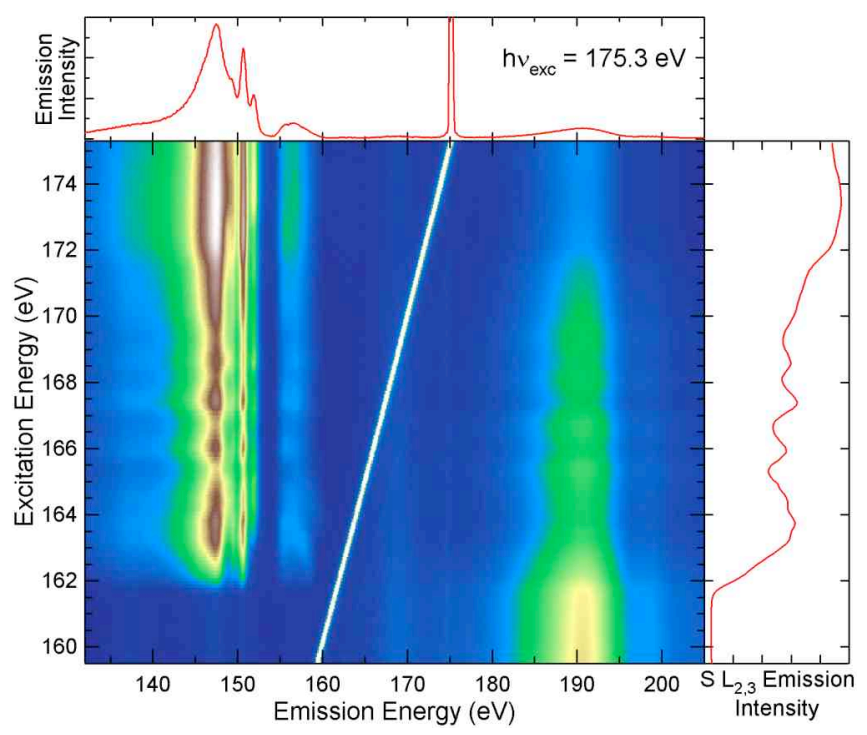

Weinhardt et al. Figure 1 


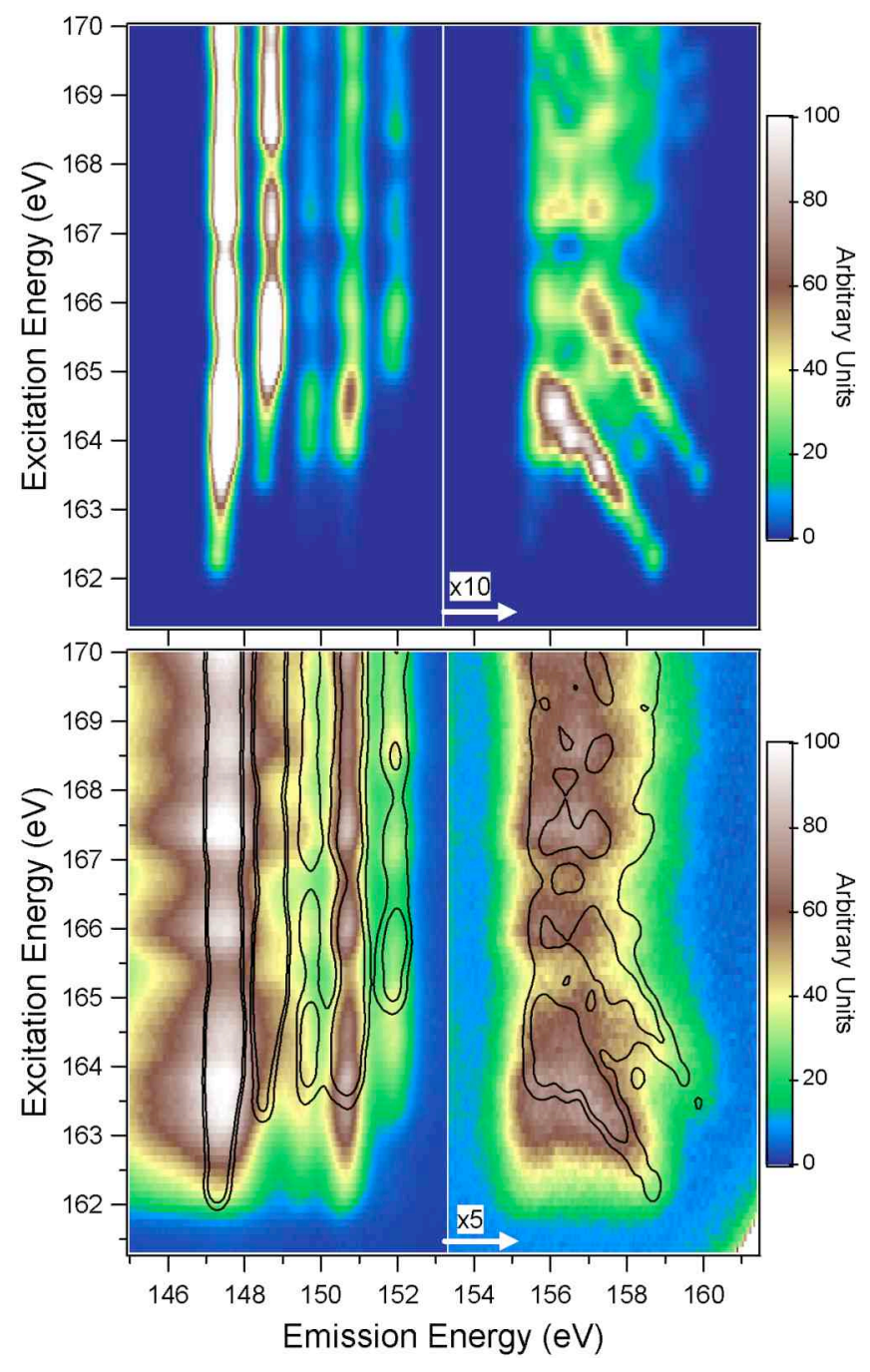

Weinhardt et al., Figure 2 


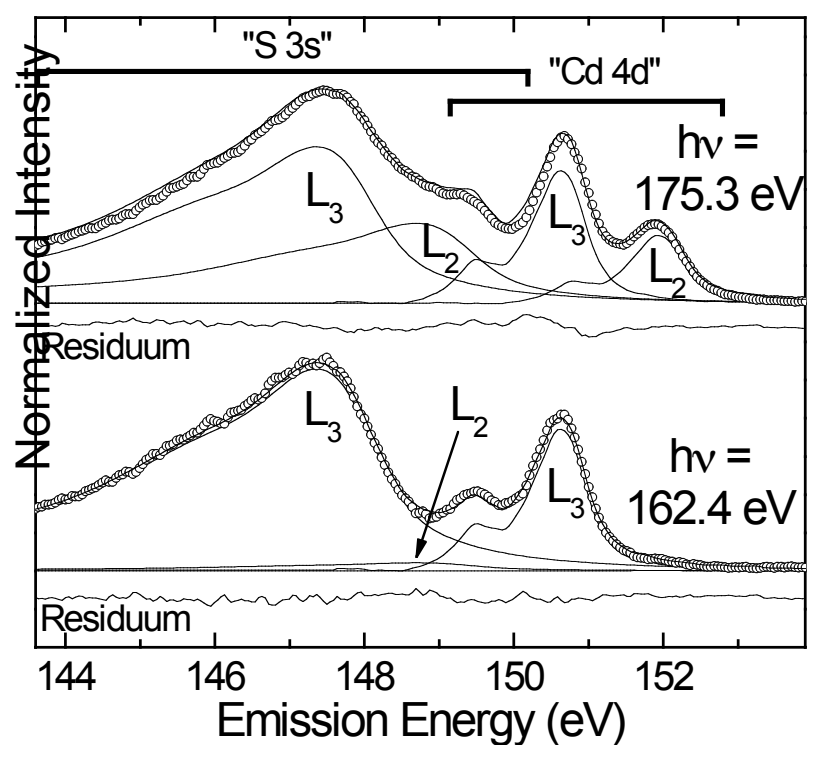

Weinhardt et al., Figure 3 


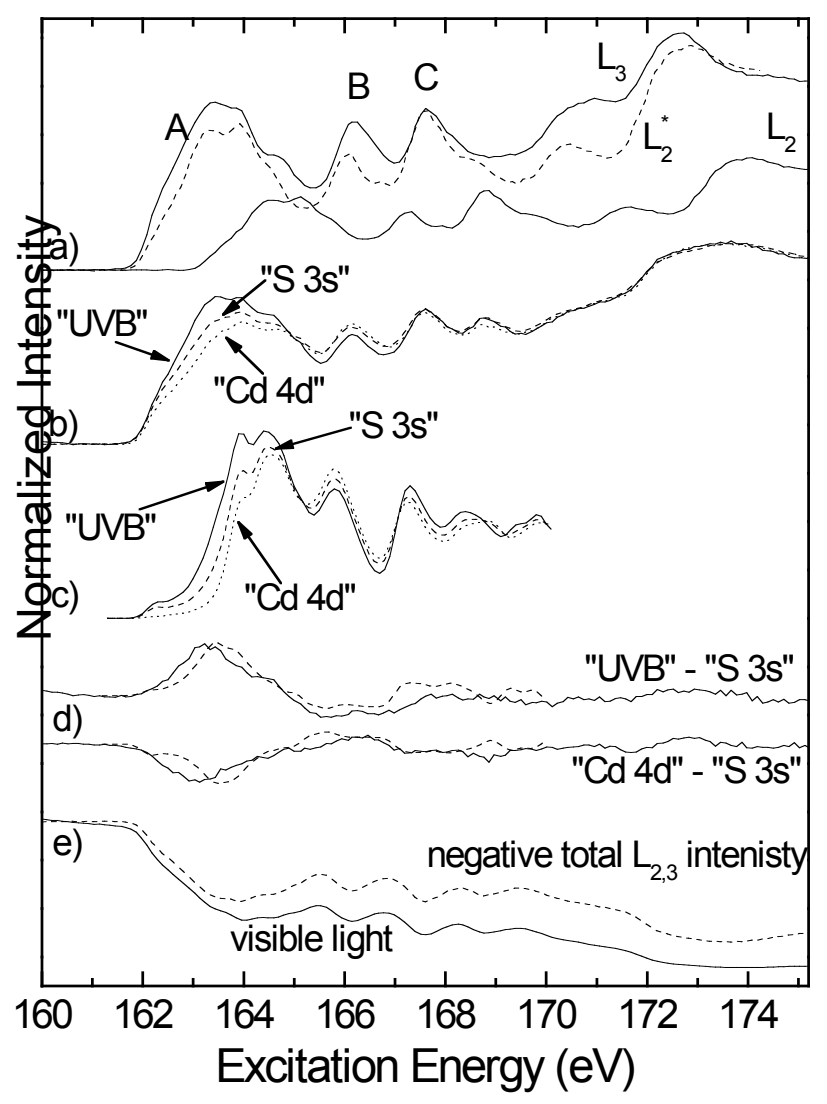

Weinhardt et al., Figure 4 